\title{
Is there a place for pudendal nerve maximal electrical stimulation for the treatment of detrusor hyperreflexia in spinal cord injury patients?
}

\author{
JG Prévinaire ${ }^{1}, \mathrm{JM}_{\text {Soler }}{ }^{2}$ and M Perrigot ${ }^{3}$ \\ ${ }^{1}$ Centre de Rééducation Fonctionelle L’Espoir, 25 Pavé du Moulin BP1, 59260 Hellemmes; ${ }^{2}$ Centre Bouffard-Vercelli, \\ Cerbère; ${ }^{3}$ Hôpital Pitié-Salpétrière, Paris, France
}

\begin{abstract}
The aim of the study is to determine whether pudendal nerve maximal electrical stimulation (MES) could represent an alternative treatment for detrusor hyperreflexia in spinal cord injury (SCI) patients. Six suprasacral SCI patients participated in the study. The treatment consisted of daily stimulation periods of $20 \mathrm{~min}$, repeated five times a week, during 4 weeks, with continuous electrical stimulation of the penis or of the clitoris via bipolar surface electrodes (rectangular stimuli of $0.5 \mathrm{~ms}$ pulse duration, $5 \mathrm{~Hz}$ frequency), with the maximum tolerable stimulation strength (under the level of pain). In two patients, additional stimulation was administrated by means of an anal plug during the last 2 weeks. The stimulus strengths ranged from 35 to $99 \mathrm{~mA}$ (mean $54 \mathrm{~mA}$ ). One patient stopped MES after 2 weeks. At the end of the treatment, neither the cystometric bladder capacities $(153 \mathrm{ml} v \mathrm{~s} 157 \mathrm{ml})$ nor the micturition charts had significantly improved for the five remaining patients. Only two patients experienced non lasting improvement of nocturia at some time of the treatment. In conclusion, we were not able to demonstrate the efficacy of MES in inhibiting detrusor hyperreflexia in SCI patients. To reach therapeutic effects, other parameters may be needed, such as higher stimulation strengths (currents above or equal to $99 \mathrm{~mA}$ ) or other currents (such as interferential therapy). Chronic stimulation with external or implanted electrodes using lower currents may represent an alternative.
\end{abstract}

Keywords: electrical stimulation; pudendal nerve; detrusor hyperreflexia; spinal cord injury patients

\section{Introduction}

Experimental studies in spinal cord injury (SCI) patients have demonstrated the efficacy of perineal electrical stimulation (ES) in short-term bladder inhibition. $^{1-4}$ Studies in the $1970 \mathrm{~s}$ stated that pudendal nerve maximal electrical stimulation (MES) could induce a profound and long-lasting bladder inhibition in SCI patients. ${ }^{5-7}$ These results, essentially based upon clinical observations without statistical validation, were never confirmed.

The aim of this study is to determine whether pudendal nerve MES could represent an alternative treatment for detrusor hyperreflexia in SCI patients. In a preliminary study in ten chronic SCI patients, we confirmed the efficacy of pudendal nerve ES in bladder inhibition. ${ }^{4}$ We also stressed the importance of current strength, as this inhibition was only achieved with the highest current strengths (ie twice the bulbocavernosus reflex threshold). These results represent the experimental background for the

Correspondence: JG Prévinaire, Centre Hélène Borel, 59283 Raimbeaucort, France therapeutic use of MES. In this study, the effect of MES on detrusor hyperreflexia in six chronic suprasacral SCI patients is reported.

\section{Methods}

Six chronic suprasacral SCI patients, five males and one woman, were treated by MES (Table 1). The selection of the patients was based on the presence of detrusor hyperreflexia confirmed by previous urodynamic studies. They all presented detrusor-sphincter dyssynergia. Reflex voiding was the method of bladder emptying in five patients, combined with clean intermittent catheterization (CIC) in four. CIC alone was used in one. All male patients were fitted with external collecting devices. None of the six patients had any significant urological history. Any medication for bladder dysfunction was stopped at least $72 \mathrm{~h}$ before testing. No patient had symptomatic urinary tract infection at the start of the study. They all received prophylactic antibiotic during the stimulation period.

Urodynamic investigations were conducted on entering the study and 4 weeks later. Patients were 
Table 1 Characteristics of patients

\begin{tabular}{lc}
\hline Sex ratio & $5 \mathrm{M} / 1 \mathrm{~F}$ \\
Age at injury & \\
Mean \pm SD & 41.7 years \pm 13.0 \\
Range & $18-57$ years \\
Time since injury & \\
Mean \pm SD & 9.3 months \pm 5.5 \\
Range & $6-20$ months \\
ASIA impairment scale & \\
Tetraplegia, incomplete (B) & 3 \\
Tetraplegia, incomplete (C) & 1 \\
Paraplegia, incomplete (B) & 2 \\
Voiding modes & 1 \\
Reflex voiding alone & 4 \\
Reflex voiding and CIC & 1 \\
CIC alone & \\
\hline
\end{tabular}

asked to record micturition frequency, voided volumes and incidental leakage of urine during $48 \mathrm{~h}$ periods before the start and throughout MES treatment.

Cystometries were performed as previously described. ${ }^{4}$ The water cystometries were done at a filling rate of $50 \mathrm{ml} / \mathrm{min}$ using a double lumen, transurethral $8 \mathrm{~F}$ Porges catheter. Perineal electromyography using a concentric needle was also recorded during the cystometry, but rectal and urethral pressures were not systematically measured. The filling was stopped at the onset of an uninhibited bladder contraction of more than $15 \mathrm{~cm}$ $\mathrm{H}_{2} \mathrm{O}$.

The treatment consisted of daily stimulation periods of $20 \mathrm{~min}$, repeated five times a week, during 4 weeks. MES was applied to the dorsal penile nerve with Velcro ribbon electrodes (Dantec) and to the dorsal nerve of the clitoris with a bipolar surface disc electrode (Dantec). A continuous biphasic current with repetitive stimulation $(5 \mathrm{~Hz})$ and rectangular stimuli ( $0.5 \mathrm{~ms}$ duration) was conducted with a Dantec Contrepoint EMG system. The individual maximal tolerable stimulation strength was chosen by slowly increasing the current intensity (with a maximum of $99 \mathrm{~mA}$ delivered by the stimulation device), and readjusted on a daily basis. Additional ES by means of an anal plug was performed in two patients (JV, TS) for the last 2 weeks of treatment. In these cases, pudendal and anal ES increased in parallel.

Discontinuous, non invasive cardiovascular monitoring was performed during the cystometries and the stimulation periods. All subjects were fully informed about the experimental procedures and gave their informed consent. They all volunteered for this protocol approved by the local ethical committee. Cystometric data (bladder capacity) were analyzed with a Wilcoxon test using paired data. A significance level of 0.05 was adopted. Changes in the micturition charts were analyzed on a case by case basis.
Table 2 Intensities of ES and cystometric bladder capacities before $(\mathrm{BC} 1)$ and after $(\mathrm{BC} 2) \mathrm{MES}$

\begin{tabular}{lccc}
\hline Patients & $\begin{array}{c}\text { Intensity } \\
(\mathrm{mA})\end{array}$ & $\begin{array}{c}B C l \\
(\mathrm{ml})\end{array}$ & $\begin{array}{c}B C 2 \\
(\mathrm{ml})\end{array}$ \\
\hline GS & 40 & 185 & 155 \\
JV & 35 & 140 & 75 \\
TS & 35 & 50 & 40 \\
VV & 75 & 250 & 290 \\
VJ & 40 & 140 & 225 \\
DC & 99 & $(270)$ & - \\
Mean & 54,0 & 153.0 & $157.0^{\mathrm{a}}$ \\
SD & \pm 26.8 & \pm 73.1 & \pm 103.4 \\
\hline
\end{tabular}

${ }^{\mathrm{a}} \mathrm{NS}(P=0.89)$

\section{Results}

Table 2 shows the results of the study. The current amplitudes varied widely between patients, but were relatively constant for each patient during the 20 sessions. Stimulation strengths ranged from 35 to $99 \mathrm{~mA}$ (mean $54.0 \mathrm{~mA}$ ). Pelvic floor muscle contractions were seen in all. One patient (DC, tetraplegia, ASIA Impairment Scale B) dropped out of the study after 15 days due to a prostatitis. In this case, we only analyzed the micturition chart for the 10 first days of treatment.

For the five remaining patients, the stimulation strengths ranged from 35 to $65 \mathrm{~mA}$ (mean $41.0 \mathrm{~mA}$ ). There were no statistical differences between the mean cystometric bladder capacity values before and after treatment $(153 \mathrm{ml}$ and $157 \mathrm{ml}$, respectively). The analysis of the micturition charts did not reveal any improvement in micturition frequency or voided volumes in any patients. Only two patients (GS, TS) experienced non-lasting improvement of nocturia at some time of the stimulation period.

The MES was well tolerated in all patients. The individual maximum tolerable stimulus was occasionally described as unpleasant, but never as painful. No changes in blood pressure or pulse rate were noted during the stimulation periods.

\section{Discussion}

In non-neuropathic bladder dysfunction, experimental and clinical studies have shown that maximal electrical stimulation (MES) induces pronounced and lasting bladder inhibition. ${ }^{8}$ Limited experience exists in its use in the treatment of neuropathic bladder dysfunction (Table 3). 5,6,9-11 Former studies stated that MES resulted in an improvement of detrusor hyperreflexia in SCI patients. ${ }^{5-7}$ From our clinical and cystometric evaluations, we were not able to demonstrate the efficacy of MES in inhibiting detrusor hyperreflexia in SCI patients.

Micturition chart recordings and cystometric bladder capacity evaluations were chosen to record 
Table 3 MES for neuropathic bladder dysfunctions

\begin{tabular}{|c|c|c|c|c|c|c|c|c|}
\hline Authors & Patients & Site of ES & Current & Frequency & $\begin{array}{l}\text { Pulse } \\
\text { duration }\end{array}$ & Intensity & Protocol & Improvement \\
\hline $\begin{array}{l}\text { Godec } \\
1979^{5}\end{array}$ & SCI & $\begin{array}{l}\text { Anal and } \\
\text { Levator ani }\end{array}$ & $\begin{array}{l}\text { Continuous } \\
\text { Monoph }\end{array}$ & $20 \mathrm{~Hz}$ & $1.0 \mathrm{~ms}$ & $\begin{array}{c}100-150 \\
\mathrm{~mA}\end{array}$ & $\begin{array}{c}4-10 \text { sessions of } 20^{\prime} \\
\text { Every } 2-3 \text { days }\end{array}$ & Yes \\
\hline $\begin{array}{l}\text { Plevnik } \\
1979^{6}\end{array}$ & $\mathrm{SCI}$ & Anal & $\begin{array}{l}\text { Continuous } \\
\text { Monoph }\end{array}$ & $20 \mathrm{~Hz}$ & $1.0 \mathrm{~ms}$ & $\begin{array}{l}30-100 \\
\mathrm{~mA}\end{array}$ & $\begin{array}{l}1 \text { session of } 20^{\prime} \\
\text { One day }\end{array}$ & Yes \\
\hline $\begin{array}{l}\text { Ohlsson } \\
1989^{9}\end{array}$ & $\begin{array}{l}\text { not } \\
\text { defin }\end{array}$ & $\begin{array}{c}\text { Anal and } \\
\text { vaginal }\end{array}$ & $\begin{array}{l}\text { Continuous } \\
\text { Altern }\end{array}$ & $10 \mathrm{~Hz}$ & $1.0 \mathrm{~ms}$ & $2-120 \mathrm{~mA}$ & $\begin{array}{c}4 \text { sessions of } 20^{\prime} \\
\text { Each week }\end{array}$ & Yes \\
\hline $\begin{array}{l}\text { Petersen } \\
1994^{10}\end{array}$ & MS & Anal & $\begin{array}{l}\text { Intermittent } \\
\text { Monoph }\end{array}$ & $40 \mathrm{~Hz}$ & $0.3 \mathrm{~ms}$ & $\begin{array}{l}\text { pain } \\
\text { threshold }\end{array}$ & $\begin{array}{l}24 \text { sessions of } 30^{\prime} \\
\text { Twice daily }\end{array}$ & No \\
\hline $\begin{array}{l}\text { Primus } \\
1996^{11}\end{array}$ & MS & $\begin{array}{l}\text { Anal or } \\
\text { vaginal }\end{array}$ & $\begin{array}{l}\text { Intermittent } \\
\text { Monoph }\end{array}$ & $20 \mathrm{~Hz}$ & $1.0 \mathrm{~ms}$ & $95 \mathrm{~mA}$ & $\begin{array}{l}15 \text { sessions of } 20^{\prime} \\
5 \text { days per week }\end{array}$ & Yes \\
\hline Present study & SCI & $\begin{array}{l}\text { Penis or } \\
\text { clitoris }\end{array}$ & $\begin{array}{l}\text { Continuous } \\
\text { Altern }\end{array}$ & $5 \mathrm{~Hz}$ & $0.5 \mathrm{~ms}$ & $35-99 \mathrm{~mA}$ & $\begin{array}{l}20 \text { sessions of } 20^{\prime} \\
5 \text { days per week }\end{array}$ & No \\
\hline
\end{tabular}

therapeutic effects. The cystometric bladder capacity is reproducible in chronic SCI patients, ${ }^{12}$ but may not be the most appropriate instrument to record changes in an overactive bladder. ${ }^{9,13}$ On the other hand, the micturition chart, that reflects better the normal life conditions, provides more valuable information." Electrical parameters used in this study were in line with general recommendations to achieve bladder inhibition. They were discussed in our preliminary paper. ${ }^{4}$

Most authors feel that the strongest electrical stimulation (ES) has the highest probability to be efficient. ${ }^{8,13}$ In the uninhibited overactive bladder, Ohlsson et al found a clear correlation between the current intensity and the clinical result. ${ }^{9}$ In this study, the current amplitudes were adjusted just below the pain threshold, but varied widely between patients (Table 2). The relatively low mean intensities found in our patients are a possible explanation for the failure of the treatment. Additional anal ES was applied in two patients, so that this low intensity could be compensated for by a higher degree of afferent pudendal nerve activation, ${ }^{14}$ but this failed to induce any change. If higher intensities are needed to reach a therapeutic effect, a selection of patients should be made, as this MES could only be tolerated by few SCI patients, mainly by patients with a complete lesion (ASIA Impairment Scale A).

The frequency of ES is another parameter to consider. Low-frequency stimulation of $5 \mathrm{~Hz}$ is optimal for bladder inhibition whereas high-frequency stimulation of $50 \mathrm{~Hz}$ is optimal to achieve the urethral closure. $^{8}$ The low frequency of $5 \mathrm{~Hz}$ is unpleasant. Subjects are able to discriminate each single pulse. In further studies in complete or incomplete SCI patients, the use of a better tolerated 10 to $20 \mathrm{~Hz}$ frequency ES seems a reasonable compromise between optimal bladder inhibition and patient tolerance. ${ }^{8}$ Petersen et al failed to show any evidence of acute or chronic bladder inhibition by sphincter stimulation in multiple sclerosis patients. ${ }^{10}$

Table 3 shows differences in other electrical parameters. However, the advantage of monophasic $v s$ biphasic or continuous $v s$ alternating currents has not yet been demonstrated. Different stimulation sites can be used, but ES of nonmuscular somatic afferent branches of the pudendal nerve (dorsal penile nerve and dorsal nerve of the clitoris) could provide a more specific bladder inhibition than other available techniques. ${ }^{2,3}$ The relatively short interval between spinal cord injury and electrical stimulation (Table 2) could raise the question as to the progress of the neuropathic voiding dysfunctions. However, the follow-up cystometries performed later revealed that the patients were all stabilised at the time of the study. The discussion on the possible mechanisms of action can be found in our preliminary paper. ${ }^{4}$

In conclusion, we were not able to demonstrate the efficacy of pudendal nerve MES in inhibiting detrusor hyperreflexia in SCI patients. To reach therapeutic effects, currents above or equal to $99 \mathrm{~mA}$ may be needed. If so, MES treatment will only be applicable to a few patients. Other types of currents, such as interferential therapy used in multiple sclerosis patients, ${ }^{15}$ are worth trying. Chronic ES with external or implanted electrodes using lower currents may represent an alternative. ${ }^{16,17}$

\section{Acknowledgements}

This work was supported by a grant from the INSERM (921106). 


\section{References}

1 Vereecken RL, Das J, Grisar P. Electrical sphincter stimulation in the treatment of detrusor hyperreflexia of paraplegics. Neurourol Urodyn 1984; 3: 145-154.

2 Vodusek DB, Light JK, Libby JM. Detrusor inhibition induced by stimulation of pudendal nerve afferents. Neurourol Urodyn 1986; 5: $381-389$.

3 Wheeler JS, Walter JS, Zaszczurynski PJ. Bladder inhibition by penile nerve stimulation in spinal cord injury patients. $J$ Urol 1992; 147: $100-103$.

4 Prévinaire JG et al. Short-term effect of pudendal nerve electrical stimulation on detrusor hyperreflexia in spinal cord injury patients: importance of current strength. Paraplegia 1996; 34: $95-99$.

5 Godec C, Cass AS. Electrical stimulation for voiding dysfunction after spinal cord injury. J Urol 1979; 121: $73-75$.

6 Plelvnik S, Janez J. Maximal electrical stimulation for urinary incontinence. Urology 1979; 14: 638-645.

7 Merrill DC. The treatment of detrusor incontinence by electrical stimulation. J Urol 1979; 122: 515-517.

8 Fall M, Lindström S. Electrical stimulation: a physiologic approach to the treatment of urinary incontinence. Urol Clin North Amer 1991; 18: 393 - 407.

9 Ohlsson BL, Fall M, Frankenberg-Sommar S. Effects of external and direct pudendal nerve maximal electrical stimulation in the treatment of the uninhibited overactive bladder. Br J Urol 1989; 64: $374-380$.
10 Petersen $\mathrm{T}$ et al. Anal sphincter maximal functional electrical stimulation in detrusor hyperreflexia. J Urol 1994; 152: $1460-$ 1462.

11 Primus G, Kramer G. Maximal external electrical stimulation for treatment of neurogenic or non-neurogenic urgency and/or urge incontinence. Neurourol Urodyn 1996; 15: 187-194.

12 Soler JM et al. Reproductibilité des cystomanométries et sphinctérométries à eau des blessés vertébro-médullaires. A propos de 30 cas. SIFUD Cannes, 1989; 2: 146-149.

13 Plevnik S, Homan G, Vrtacnik P. Short-term maximal electrical stimulation for urinary retention. Urology 1984; 24: 521 - 523 .

14 Erkisen BC, Bergmann S, Eik-Nes SH. Maximal electrostimulation of the pelvic floor in female idiopathic detrusor instability and urge incontinence. Neurourol Urodyn 1989; 8: 219-230.

15 Van Poppel H, Ketelaer P, Van De Weerd A. Interferential therapy for detrusor hyperreflexia in multiple sclerosis. Urology 1985; 25: $607-612$

16 Wheeler JS, Walter JS, Sibley P. Management of incontinent SCI patients with penile stimulation: preliminary results. $J \mathrm{Am}$ Paraplegia Soc 1994; 17: 55-59.

17 Ishigooka $\mathrm{M}$ et al. Electrical pelvic floor stimulation: a possible alternative treatment for reflex urinary incontinence in patients with spinal cord injury. Spinal Cord 1996; 34: 411-415. 